\title{
MODULES DETERMINED BY THEIR TOPS AND SOCLES
}

\author{
K. BONGARTZ AND S. O. SMALØ
}

\begin{abstract}
We prove that a module in a preprojective component of the AuslanderReiten quiver of an artin algebra is determined by its top and socle. Also other invariants determining such a module are given.
\end{abstract}

Let $A$ be an artin algebra over a commutative artin ring $k$. All modules considered here will be of finite length. A connected component $\mathscr{C}$ of the Auslander-Reiten quiver (A-R-quiver) $\Gamma_{A}$ of $A$ is called a preprojective component if $\mathscr{C}$ contains no oriented cycles and if any indecomposable module $U$ belonging to $\mathscr{C}$ is isomorphic to $(\operatorname{Tr} D)^{n} P$ for some indecomposable projective module $P$ and some nonnegative number $n$ (see [5]). We prove the following result:

THEOREM. Let $M$ be an indecomposable module belonging to a preprojective component of the Auslander-Reiten quiver of $A$. Denote by $P_{1} \rightarrow P_{0} \rightarrow M \rightarrow 0$ a minimal projective resolution of $M$ and by $I_{0}$ an injective hull of $M$. Then the following conditions on an indecomposable module $N$ are equivalent:

(a) $N \simeq M$.

(b) $|\operatorname{Hom}(Q, N)|=|\operatorname{Hom}(Q, M)|$ for any projective module $Q$. (Here $|X|$ denotes the length of $X$ as a $k$-module.)

(c) $N$ has a minimal projective resolution $P_{1} \rightarrow P_{0} \rightarrow N \rightarrow 0$.

(d) $P_{0}$ is a projective cover of $N$ and $I_{0}$ is an injective hull of $N$.

Results along this line have been obtained by several persons (see [2-6]). That (b) implies (a) follows from [6], but for the convenience of the reader we include an argument for this. The other two implications seem to be new even though special cases are included in [2-4].

Before we start with the proof of the theorem, we recall some facts which we use later on without comments. It is easy to see that a component $\mathscr{C}$ of the A-R-quiver $\Gamma_{4}$ is a preprojective component if and only if $\mathscr{C}$ contains no oriented cycles and each module $X$ in $\mathscr{C}$ has the property that $\operatorname{Hom}(Y, X)$ is nonzero for only a finite number of indecomposable modules $Y$. Furthermore, if $X$ is in a preprojective component $\mathscr{C}$ and $\operatorname{Hom}(Y, X)$ is nonzero, then $Y$ is a predecessor of $X$, that means that there is a chain $Y=X_{0} \rightarrow X_{1} \rightarrow \cdots \rightarrow X_{n}=X$ of irreducible maps (see [5, §1.3]). In particular, $Y$ also belongs to the preprojective component $\mathscr{C}$.

Combining this observation with some general formulas of Auslander and Reiten one obtains the following interesting consequences which are crucial for our proof.

Received by the editors February 25, 1985.

1980 Mathematics Subject Classification. Primary 16A64, 16A46.

(C) 1986 American Mathematical Society $0002-9939 / 86 \$ 1.00+\$ .25$ per page 
Let $M$ be an indecomposable module in a preprojective component.

(1) $\operatorname{Ext}^{1}(M, M)$ is equal to zero because $\operatorname{Ext}^{1}(M, M)$ is isomorphic to

\section{$D \underline{\operatorname{Hom}}(\operatorname{Tr} D M, M)$}

(see [1, Proposition 2.2 and line 1, p. 253]) which is included in $D \operatorname{Hom}(\operatorname{Tr} D M, M$ ), and this is zero because there are no oriented cycles.

(2) If each indecomposable projective module $P$ is a predecessor of $M$, we have that $\operatorname{Hom}(\operatorname{Tr} D X, A)$ is zero for each indecomposable module $X$ with $\operatorname{Hom}(M, X)$ nonzero. Hence, we conclude that the injective dimension of such an $X$ is less than or equal to one by [2, Proposition 3.8(b)].

(3) If no indecomposable injective module is a predecessor of $D \operatorname{Tr} M$ we have that $\operatorname{Hom}(I, D \operatorname{Tr} X)$ is zero for all injective modules $I$ and all predecessors $X$ of $M$. Therefore, by [2, Proposition 3.7], the dual of the result referred to above, the projective dimension of $X$ is less than or equal to one for all predecessors $X$ of $M$.

We fix a set $\mathscr{S}$ of representatives of isomorphism classes of simple $A$-modules. For each $S$ in $\mathscr{S}$ we denote by $P(S)$ a projective cover of $S$ and $I(S)$ an injective hull of $S$. For each $A$-module $X$ and each $S$ in $\mathscr{S}$ we have that $|\operatorname{Hom}(P(S), X)|$ is equal to $|\operatorname{Hom}(X, I(S))|$.

Finally, before starting the proof of the theorem we recall some facts about the restriction functor $\operatorname{Hom}(P$,$) for P$ a projective $A$-module.

The support of a module $M$, denoted supp $M$, is the set of all $P(S)$ such that $\operatorname{Hom}(P(S), M)$ is nonzero. Let $P$ be equal to $\amalg_{\mathrm{S} \in \mathscr{T}} P(S)$ for some $\mathscr{T} \subseteq \mathscr{S}$, and let $B$ be the opposite of the ring of endomorphisms of $P, \operatorname{End}(P)^{\mathrm{op}}$.

(4) $\operatorname{Hom}(P$,$) induces an equivalence between the category of A$-modules $X$ with projective presentation $P_{1} \rightarrow P_{0} \rightarrow X \rightarrow 0$ where the indecomposable summands of $P_{0}$ and $P_{1}$ are amongst $P(S)$ with $S$ in $\mathscr{T}$, and the $B$-modules.

(5) $\operatorname{Hom}(P$,$) induces an equivalence between the category of A$-modules $X$ such that all simple summands of the top of $X$ and the socle of $X$ are in $\mathscr{T}$, and the $B$-modules. In particular, $\operatorname{Hom}(P$,$) induces a full and faithful functor from the$ subcategory of $A$-modules $X$ with the support of $X$ in $\{P(S) \mid S \in \mathscr{T}\}$ to the $B$-modules. (See [2, §1, Case (a) and (b)].)

Proof of ThE Theorem. To show that (b) implies (a), (c) implies (a) and (d) implies (a) we prove in each case that $\operatorname{Hom}(N, M)$ is different from zero, hence by the comments above, $N$ belongs to the same preprojective component as $M$. By symmetry, we then have that $\operatorname{Hom}(M, N)$ is nonzero and then, by the absence of oriented cycles, $M$ and $N$ have to be isomorphic modules.

(b) implies (a). We first reduce to the case where $\operatorname{Hom}(Q, M)$ is nonzero for aly indecomposable projective modules $Q$ by using the restriction functor $R=\operatorname{Hom}(P$, where $P$ is the direct sum of the $P(S)$ in supp $M$. Then $R(M)$ is an indecomposable $B$-module where $B=\operatorname{End}(P)^{\text {op }}$ by remark (5), and $\operatorname{Hom}(Q, R M)$ is nonzero for all indecomposable projective $B$-modules. Further, again by the equivalence in remark (5) it is not hard to see that $R M$ is in a preprojective component $\mathscr{C}$ of the A-R-quiver $\Gamma_{B}$ of $B$. Hence, we may assume $\operatorname{Hom}(Q, M)$ is nonzero for all indecomposable projective modules $Q$. Then $P(S)$ is a predecessor of $M$ for all $S$ in $\mathscr{S}$ and therefore 
$\operatorname{Hom}(\operatorname{Tr} D M, A)$ is zero, which by remark (2) implies that there is an injective resolution $0 \rightarrow M \rightarrow I_{0} \rightarrow I_{1} \rightarrow 0$ of $M$. Applying $\operatorname{Hom}(M$,$) to this sequence and$ the fact that $\operatorname{Ext}^{1}(M, M)$ is zero, we get that $|\operatorname{Hom}(M, M)|=\left|\operatorname{Hom}\left(M, I_{0}\right)\right|-$ $\left|\operatorname{Hom}\left(M, I_{1}\right)\right|$. Similarly, applying $\operatorname{Hom}(N$,$) to the same sequence and using the fact$ that $|\operatorname{Hom}(M, I)|=|\operatorname{Hom}(N, I)|$ for all injective modules $I$, we get that

$$
\begin{aligned}
|\operatorname{Hom}(N, M)| & =\left|\operatorname{Hom}\left(N, I_{0}\right)\right|-\left|\operatorname{Hom}\left(N, I_{1}\right)\right|+\left|\operatorname{Ext}^{1}(N, M)\right| \\
& \geqslant\left|\operatorname{Hom}\left(N, I_{0}\right)\right|-\left|\operatorname{Hom}\left(N, I_{1}\right)\right| \\
& =\left|\operatorname{Hom}\left(M, I_{0}\right)\right|-\left|\operatorname{Hom}\left(M, I_{1}\right)\right| \\
& =|\operatorname{Hom}(M, M)| \geqslant 1
\end{aligned}
$$

which completes the proof of this implication.

(c) implies (a). This implication is obvious if $M$ is a projective module. Therefore, we may assume that $M$ is a nonprojective module. Then consider the almost split sequence

$$
0 \rightarrow D \operatorname{Tr} M \rightarrow X \rightarrow M \rightarrow 0
$$

ending in $M$. Now let $P$ be the direct sum of those $P(S)$ which are in the support of $X$. Because the top of $P_{1}$ is isomorphic to the socle of $D \operatorname{Tr} M$ (see e.g. [1, Proposition 5.3]), all indecomposable direct summands of $P_{1}$ occur as summands of $P$. Denote by $R$ the restriction functor $\operatorname{Hom}(P$,$) from A$-modules to $B$-modules where $B=\operatorname{End}(P)^{\mathrm{op}}$. We obtain

$$
R P_{1} \rightarrow R P_{0} \rightarrow R M \rightarrow 0 \text { and } R P_{1} \rightarrow R P_{0} \rightarrow R N \rightarrow 0
$$

as the first terms of a minimal projective resolution of $R M$ and $R N$, respectively. From remark (5) we have that

$$
0 \rightarrow R D \operatorname{Tr} M \rightarrow R X \rightarrow R M \rightarrow 0
$$

is an almost split sequence of $B$-modules. Since none of the indecomposable projective $B$-modules belong to cycles of nonisomorphisms between indecomposable $B$-modules and all indecomposable projective $B$-modules have nonzero homomorphisms to $R X$, the component $\mathscr{C}$ of the A-R-quiver $\Gamma_{B}$ of $B$ containing $R M$ is a preprojective component.

Further, since $\operatorname{Hom}(Q, R X)$ is nonzero for all indecomposable projective $B$-modules $Q$, we have that $\operatorname{Hom}(R X, I)$ is nonzero for all indecomposable injective $B$-modules $I$. Hence, $\operatorname{Hom}(J, D \operatorname{Tr} M)$ is zero for all indecomposable injective $B$-modules $J$ and therefore by remark (3) above

$$
0 \rightarrow R P_{1} \rightarrow R P_{0} \rightarrow R M \rightarrow 0
$$

is exact.

From this reduction we may as well assume from the beginning that

$$
0 \rightarrow P_{1} \rightarrow P_{0} \rightarrow M \rightarrow 0
$$

is exact. Applying $\operatorname{Hom}(, M)$ to this exact sequence and the exact sequence $P_{1} \rightarrow P_{0} \rightarrow N \rightarrow 0$ and using the fact that $\operatorname{Ext}^{1}(M, M)=0$ we obtain that

$$
\begin{aligned}
|\operatorname{Hom}(N, M)| & \geqslant\left|\operatorname{Hom}\left(P_{0}, M\right)\right|-\left|\operatorname{Hom}\left(P_{1}, M\right)\right| \\
& =|\operatorname{Hom}(M, M)| \geqslant 1 .
\end{aligned}
$$

This completes the proof of this implication. 
(d) implies (a). Now let $P$ be the direct sum of the $P(S)$ when $\mathscr{S}$ is isomorphic to a direct summand of either the socle of $M$ or the top of $M$.

Using remark (5) we may assume that all simple modules occur as a composition factor of either the socle of $M$ or the top of $M$. In the absence of cycles, $S$ is divided into two disjoint subsets

$$
\mathscr{X}=\{T \mid \operatorname{Hom}(M, T) \neq 0\} \quad \text { and } \quad \mathscr{Y}=\{S \mid \operatorname{Hom}(S, M) \neq 0\} .
$$

By remarks (2) and (3) each $T$ in $\mathscr{X}$ has injective dimension less than or equal to one and each $S$ in $Y$ has projective dimension less than or equal to one. Moreover, the relation $S_{1} \leqslant S_{2}$ if $\operatorname{Ext}^{1}\left(S_{1}, S_{2}\right)$ is nonzero, generates a partial ordering $\leqslant$ on $\mathscr{S}$ since all the indecomposable projective modules belong to the preprojective component $\mathscr{C}$ containing $M$.

First, we prove the equality

$$
|\operatorname{Hom}(P(S), M)|=|\operatorname{Hom}(P(S), N)| \quad \text { for all } S \in \mathscr{Y}
$$

by induction on $\leqslant$. The statement is clear for minimal $S$ because $P(S)$ is then isomorphic to $S$.

Each $S \in \mathscr{Y}$ has a minimal projective resolution $0 \rightarrow \overline{P(S)} \rightarrow P(S) \rightarrow S \rightarrow 0$ where $\overline{P(S)}$ is a direct sum of $P\left(S^{\prime}\right)$ with $S^{\prime}<S$. We obtain exact sequences

$$
0 \rightarrow \operatorname{Hom}(S, M) \rightarrow \operatorname{Hom}(P(S), M) \rightarrow \operatorname{Hom}(\overline{P(S)}, M) \rightarrow \operatorname{Ext}^{1}(S, M)
$$

and

$$
0 \rightarrow \operatorname{Hom}(S, N) \rightarrow \operatorname{Hom}(P(S), N) \rightarrow \operatorname{Hom}(\overline{P(S)}, N) \rightarrow \operatorname{Ext}^{1}(S, N) .
$$

The end terms have to be zero because $S$, being a predecessor of $M$, does not lie on an oriented cycle. Induction applies.

Similarly, we show the inequality $|\operatorname{Hom}(M, I(T))| \geqslant|\operatorname{Hom}(N, I(T))|$ for any $T \in \mathscr{X}$. This is obvious if $T$ is maximal in $\mathscr{X}$. Let $0 \rightarrow T \rightarrow I(T) \rightarrow \overline{I(T)} \rightarrow 0$ be an injective resolution of $T \in \mathscr{X}$. Then $\overline{I(T)}$ is a direct sum of some $I\left(T^{\prime}\right)$ satisfying $T<T^{\prime}$. An easy calculation gives the following inequality:

$$
\begin{aligned}
\mid \operatorname{Hom}(M, I(T) \mid & =|\operatorname{Hom}(M, T)|+\mid \operatorname{Hom}(M, \overline{I(T)} \mid \\
& \geqslant|\operatorname{Hom}(N, T)|+\mid \operatorname{Hom}(N, \overline{I(T)} \mid \\
& =\mid \operatorname{Hom}\left(N, I(T)|+| \operatorname{Ext}^{1}(N, T) \mid\right. \\
& \geqslant|\operatorname{Hom}(N, I(T))| .
\end{aligned}
$$

Using remark (2) once more, $M$ has a minimal injective resolution $0 \rightarrow M \rightarrow I_{0}$ $\rightarrow I_{1} \rightarrow 0$. We apply $\operatorname{Hom}(M$,$) and \operatorname{Hom}(N$,$) to it and, using that |\operatorname{Hom}(P(S), X)|$ $=\mid \operatorname{Hom}(X, I(S) \mid$ for all modules $X$, we get the following inequality:

$$
\begin{aligned}
|\operatorname{Hom}(N, M)| & \geqslant\left|\operatorname{Hom}\left(N, I_{0}\right)\right|-\left|\operatorname{Hom}\left(N, I_{1}\right)\right| \\
& =\left|\operatorname{Hom}\left(M, I_{0}\right)\right|-\left|\operatorname{Hom}\left(N, I_{1}\right)\right| \\
& \geqslant\left|\operatorname{Hom}\left(M, I_{0}\right)\right|-\left|\operatorname{Hom}\left(M, I_{1}\right)\right| \\
& =|\operatorname{Hom}(M, M)|>0 .
\end{aligned}
$$

The proof of the theorem is complete. 


\section{REFERENCES}

1. M. Auslander and I. Reiten, Representation theory of Artin algebras III. Almost split sequences, Comm. Algebra 3 (1975), 239-294.

2. __ Modules determined by their composition factors, Illinois J. Math. 29 (1985), 280-301.

3. K. Bongartz, Quadratic forms and finite representation type, Séminaire d'algèbre (Malliavin), Lecture Notes in Math., Springer-Verlag (to appear).

4. D. Happel, Composition factors for indecomposable modules, Proc. Amer. Math. Soc. 86 (1982), 29-31.

5. D. Happel and C. Ringel, Tilted algebras, Trans. Amer. Math. Soc. 274 (1982), 399-443.

6. C. Ringel, Indecomposable representations of finite dimensional algebras, Report ICM 82, PWN, Warszawa, 1983.

Gesamthochschule Wuppertal, Fachbereich 7 Mathematik, Gaussstr. 205000 Wuppertal, FEDERAL RePublic OF GeRMANY

Universitetet I Trondheim, AVH, Institutt for Matematikk og StatistikK, 7055 Dragvoll, NORWAY 